\title{
Prevalence of Mental Disorders and Profile of Disablement among Primary Health Care Service Users in Lagos Island
}

\author{
V. O. Lasebikan, ${ }^{1}$ A. Ejidokun, ${ }^{2}$ and O. A. Coker $^{3}$ \\ ${ }^{1}$ Department of Psychiatry, College of Medicine, University of Ibadan, PMB 5116, Ibadan, Nigeria \\ ${ }^{2}$ School of Psychiatric Nursing, Federal Neuropsychiatric Hospital, P.O. Box 1912, Sabo, Yaba, Lagos, Nigeria \\ ${ }^{3}$ Department of Psychiatry, Lagos State University Teaching Hospital, c/o P.O. Box 1912, Sabo, Yaba, Lagos, Nigeria
}

Correspondence should be addressed to V. O. Lasebikan, victorlash@yahoo.com

Received 18 December 2011; Accepted 13 January 2012

Academic Editor: Huibert Burger

Copyright (C) 2012 V. O. Lasebikan et al. This is an open access article distributed under the Creative Commons Attribution License, which permits unrestricted use, distribution, and reproduction in any medium, provided the original work is properly cited.

Background. The aim of this study was to determine the prevalence of psychiatric morbidity in selected semiurban primary care centers in Lagos Island, Nigeria using the screening tool GHQ-12 and the ICD 10 mental disorders checklist (primary care) (ICD 10 PC). Methods. In this multistage cross-sectional study, 400 participants were recruited by using proportional sampling of 17,787 attendees. Results. Of all respondents, $45.8 \%$ scored positive on GHQ-12. The most prevalent "any ICD 10 disorder" was unexplained somatic disorder $(57.5 \%)$, while 2.0 to $7.8 \%$ of the respondents reported varying levels of disablement. Younger age $(P<0.001)$ and being widowed $(P=0.03)$ were significantly associated with high GHQ scores while younger age $(P<0.001)$ and male gender $(P=0.04)$ were significantly associated with "any ICD 10 disorder". Conclusion. These findings are a useful guide to the probable prevalence of psychiatric morbidity in primary care in Nigeria and in the design of appropriate interventions.

\section{Introduction}

The majority of individuals with psychiatric morbidity in the community are usually seen at the primary care level [1$5]$, with prevalence rates ranging from 16 to $43 \%$ of general practice users $[1,6-8]$.

In different countries of the world, the prevalence of mental disorders in primary care settings has been extensively researched and, in adults, ranges between $10 \%$ and $60 \%[8,9]$. The most prevalent mental disorders presenting in primary care settings are depression, $5 \%$ to $20 \%$ [10], generalized anxiety disorder, $4 \%$ to $15 \%$ [11], harmful alcohol use and dependence, $5 \%$ to $15 \%$ [12], and somatization disorders, $5 \%$ to $11 \%$ [13].

Studies of children and adolescents have also demonstrated a high prevalence of mental disorders in primary care settings. For example, about $20 \%$ of children and adolescent were found to have mental health problems in Ibadan, Nigeria [14], 30\% of children aged 6 to 11 years in Valencia, Spain [15], and 43\% of children aged 6 to 18 years in United Arab Emirates [16].
The prevalence of mental disorders could be as high as $33 \%$ in the elderly age group, seen in primary care $[17,18]$. The commonest diagnoses are depression and dementia [19].

Several studies in primary care facilities have measured the prevalence of other specific mental disorders, due to their public health importance. The prevalence of postnatal depression was $14 \%$ in Turkey [20] and 19\% in Nigeria [21].

Despite this high prevalence, primary care practitioners have difficulties in detecting about one-third of those with mental health problems $[4,22,23]$. This can be adduced to a number of reasons, such as reports indicating that patients seeking treatment in primary care tend to somatize their emotional distress, thereby presenting with physical symptoms rather than overt psychological symptoms [24]. Furthermore, medical history is often taken with complacency and little confidentiality thereby discouraging patients from sharing sensitive aspects of their psychological/emotional distress [6]. Also, primary care practitioners have difficulties in diagnosing and treating psychiatric disorders $[25,26]$ and often have limited time to obtain a psychiatric history [26]. 
Thus, screening for psychiatric disorders in primary care can improve the detection rate and may be helpful in preventing grave consequences of unrecognized and untreated psychiatric morbidity. This is relevant to the Nigerian setting where mental health service at the primary care has received little attention.

The main objective of the present study was to determine the prevalence and sociodemographic correlates of mental disorders among primary health care service users in Lagos Island.

\section{Methodology}

The study was a descriptive survey that determined the prevalence of psychiatric morbidity and syndromes and established the relationship between the demographic variables of the study population with psychiatric morbidity among the primary health care service users in Lagos Island. The study setting was Lagos Island area of Lagos State, south west of Nigeria with an estimated population of 400,000 residents (projected 2007 population at 3\% annual increase of 1991) [27]. The target population was primary care users, aged 15 years and above, who utilized a selected number of Primary Health Care Centre of Lagos Island between January and April, 2008.

2.1. Sample Size Estimation. Minimum sample size was obtained using minimum sample size estimation table for a population of at least 10,000 persons at $5 \%$ margin error, yielding a minimum sample of 370 [28]. Thus, 400 subjects were interviewed.

2.2. Sampling Procedure. A Multistage sampling method was used.

2.2.1. First Stage. All primary health care centers were grouped into two according to the local government area they are located in, namely, Lagos Island West andLagos Island East.

(i) Lagos Island West
(a) Sura Primary Health Care Centre.
(b) City-Hall Primary Health Care Centre.
(c) Oko-awo primary Health care Centre.

(ii) Lagos Island East
(a) Olowogbowo Primary Health Care Centre.
(b) Adeniji Adele Primary Health Care Centre.
(c) Broad Street Primary Health Care Centre.

2.2.2. Second Stage. The total number of clients registered in the preceding year was obtained from the information officers for each local government. In Lagos Island East, Sura Primary Health Care Centre registered 1833 service users in preceding year; City Hall recorded 4367 service users, while Oko-awo recorded 2762 service users. In Lagos Island West, Olowogbowo recorded 2207 service users; Adeniji
Adele recorded 3523 service users and Broad Street centre recorded 3095 service users. In all 17,787 clients utilized all the primary care centers in preceding year.

2.2.3. Stage Three. Since the number of service users in each centre varied, a quota was determined for each of the primary health care centers, using proportional sampling method.

Hence, 41 clients were interviewed in Sura Primary Health Care Centre, 98 from City Hall, 62 from Oko-awo, 50 from Olowogbowo, 79 from Adeniji Adele, and 93 from Broad Street Primary Health Care Centre, respectively. The first respondent in each facility was interviewed by simple random sampling and subsequent ones one after the other until each quota was fulfilled.

\section{Procedure for Data Collection}

One stage assessment was used in the data collection for this study. The study was introduced and briefly described to the participants at the waiting room before they entered the consulting room. Consent was also obtained from each participant.

Patients aged from 12 to 70 years. Patients were given tallies. The first subject was chosen by simple random sampling and subsequent ones by proportional sampling method.

Excluded from the study were patients who did not consent and those who were not literate.

3.1. Instruments. All respondents were administered both the GHQ-12 [29] and the ICD 10 PC [30]. The GHQ12 was used to screen for psychiatric morbidity while the Mental Health Checklist (ICD-10 PC) was used to determine prevalence of psychiatric syndromes. The respondents were also requested to complete a data form consisting of sociodemographic data, psychiatric history, and recent life events. The time taken for each respondent to be interviewed was between 12 and 20 minutes.

3.2. Ethical Considerations. Consent was obtained from each participant before the study so also was ethical approval from the Lagos State Ministry of Health Ethical Review Committee.

3.3. Analysis. Analysis was performed using SPSS software [31]. Categorical data were analyzed using person $\chi^{2}$ statistics, while continuous variables were analyzed using student $t$-test. All tests of significance were set at $95 \%$ confidence interval.

\section{Results}

The sociodemographic characteristics of the sample are shown in Table 1. A total of 400 out of an initial 470 subjects completed the interview, a response rate of $(85.1 \%)$. The sample consisted of $88(22.0 \%)$ children and young adults below aged 29 years, $55(13.8 \%)$ aged 30 to 39 years, 72 (18.0\%) aged 40 to 49 years, $63(15.8 \%)$ aged 50 to 59 years, and $122(30.5 \%)$ who were above 59 years. 
TABLE 1: Demographic data of respondents.

\begin{tabular}{|c|c|c|c|c|c|c|c|c|}
\hline Demographic characteristics & $N$ & $\%$ & $\begin{array}{c}\text { High GHQ } \\
n=182 \%\end{array}$ & $\chi^{2}$ & $P$ & $\begin{array}{c}\text { Any ICD } 10 \text { Disorder } \\
n=230 \%\end{array}$ & $\chi^{2}$ & $P$ \\
\hline \multicolumn{9}{|l|}{ Age (years) } \\
\hline$<29$ & 88 & 22.0 & 61.4 & \multirow[t]{5}{*}{11.8} & \multirow[t]{5}{*}{$<0.001$} & 79.5 & \multirow[t]{5}{*}{21.3} & \multirow[t]{5}{*}{$<0.001$} \\
\hline $30-39$ & 55 & 13.8 & 63.6 & & & 72.7 & & \\
\hline $40-49$ & 72 & 18.0 & 48.6 & & & 55.5 & & \\
\hline $50-59$ & 63 & 15.8 & 48.3 & & & 47.6 & & \\
\hline$>59$ & 122 & 30.5 & 23.8 & & & 32.8 & & \\
\hline \multicolumn{9}{|l|}{ Sex } \\
\hline Male & 174 & 43.5 & 47.1 & \multirow[t]{2}{*}{0.24} & \multirow[t]{2}{*}{0.6} & 63.2 & \multirow[t]{2}{*}{4.1} & \multirow[t]{2}{*}{0.04} \\
\hline Female & 226 & 56.5 & 44.7 & & & 53.1 & & \\
\hline \multicolumn{9}{|l|}{ Marital status } \\
\hline Married & 250 & 62.5 & 44.8 & \multirow[t]{5}{*}{10.1} & \multirow[t]{5}{*}{0.03} & 56.0 & \multirow[t]{5}{*}{5.7} & \multirow[t]{5}{*}{0.2} \\
\hline Single & 100 & 25.0 & 39.0 & & & 55.0 & & \\
\hline Divorced & 25 & 6.3 & 72.0 & & & 80.0 & & \\
\hline Separated & 17 & 4.2 & 58.8 & & & 58.8 & & \\
\hline Widow & 8 & 2.0 & 50.0 & & & 62.5 & & \\
\hline \multicolumn{9}{|l|}{ Level of education } \\
\hline No formal education & 69 & 17.3 & 49.3 & \multirow[t]{4}{*}{1.6} & \multirow[t]{4}{*}{0.7} & 58.0 & \multirow[t]{4}{*}{3.4} & \multirow[t]{4}{*}{0.3} \\
\hline Primary school & 60 & 15.0 & 50.0 & & & 66.7 & & \\
\hline Secondary school & 133 & 33.2 & 45.9 & & & 52.6 & & \\
\hline Postsecondary education & 138 & 34.5 & 42.0 & & & 57.9 & & \\
\hline \multicolumn{9}{|l|}{ Religion } \\
\hline Christianity & 188 & 47.0 & 47.9 & \multirow[t]{3}{*}{1.7} & \multirow[t]{3}{*}{0.4} & 58.5 & \multirow[t]{3}{*}{0.4} & \multirow[t]{3}{*}{0.8} \\
\hline Islam & 196 & 49.0 & 42.9 & & & 56.1 & & \\
\hline Traditional & 16 & 4.0 & 56.3 & & & 62.5 & & \\
\hline \multicolumn{9}{|l|}{ Occupation } \\
\hline High level professional & 65 & 16.3 & 44.6 & \multirow[t]{3}{*}{4.4} & \multirow[t]{3}{*}{0.2} & 61.5 & \multirow[t]{3}{*}{1.2} & 0.8 \\
\hline Skilled worker & 100 & 25.0 & 43.0 & & & 60.0 & & \\
\hline Semiskilled/unskilled & 126 & 31.5 & 40.0 & & & 55.5 & & \\
\hline Worker & & & & & & & & \\
\hline Not on any job & 109 & 27.3 & 40.4 & & & 55.0 & & \\
\hline
\end{tabular}

Of the 400 respondents, 226 (56.5\%) were females, and $250(62.5 \%)$ were married. Only 69 (17.3\%) had no formal, and $109(27.3 \%)$ were unemployed (Table 1$)$.

4.1. Psychiatric Morbidity. Prevalence of psychiatric morbidity was significantly highest for respondents below 39 years and tended to reduce with increasing age; $\chi^{2}$ for trend = 11.8, $P<0.001$. Prevalence of psychiatric morbidity was significantly highest among those who were divorced $72 \%$; $\chi^{2}=10.1, P=0.03$ (Table 1$)$.

4.2. ICD 10 Disorder. Prevalence of any ICD 10 disorder was highest among those below 29 years of age and tended to reduce with increasing age $\chi^{2}$ for trend 21.3; $P<0.001$. Prevalence of any ICD 10 disorder was significantly higher in males; $\chi^{2}=4.1, P=0.04$ (Table 1 ).

The most prevalent clinical syndrome was unexplained somatic complaints $(57.5 \%)$, followed by depression among half $(50.0 \%)$ of the respondents, any anxiety disorder (49.3\%), primary insomnia (45.3\%), and alcohol use disorder $(11.3 \%)$. Between 2.0 and $7.8 \%$ of respondents had disablement in various areas of life, out of which the commonest was in the domain of family relations $(7.8 \%)$.

Mean duration of inability to carry out activities of daily life in past month was $4.2 \pm 2.1$. Mean duration of days spent on bed in past month was $5.4 \pm 3.2$ (Table 2 ).

\section{Discussion}

The present study, aimed at determining the prevalence of psychiatric morbidity among primary health centers service users in Lagos Island, revealed a high prevalence of psychiatric morbidity of $47.8 \%$. The most prevalent ICD syndrome was unexplained somatic complaint disorder $(57.5 \%)$ while half $(50.0 \%)$ of the respondents had depressive disorder. A prevalence rate of $11.3 \%$ was reported for 
TABle 2: Clinical data of respondents.

\begin{tabular}{lcc}
\hline ICD 10 disorders & Frequency & Percentage (\%) \\
\hline Depressive episode & 200 & 50.0 \\
Any anxiety disorder & 219 & 49.3 \\
Alcohol use disorder & 45 & 11.3 \\
Primary insomnia & 223 & 45.3 \\
Fatigue problem & 23 & 5.8 \\
Unexplained somatic disorder & 230 & 57.5 \\
Limited in one or more of the & & \\
following activities in the past month & 17 & 4.3 \\
Self-care; bathing, dressing, eating & & \\
Family relation; spouse, children, & 31 & 7.8 \\
relatives & 16 & 4.0 \\
Going to school or work & 12 & 3.0 \\
Doing household work or task & 9 & 2.3 \\
Social activities; seeing friends & 8 & 2.0 \\
Remembering things & $4.2 \pm 2.1$ & - \\
Unable to carry out activities (days) & $5.4 \pm 3.2$ & - \\
Spent on bed (days) &
\end{tabular}

alcohol use disorder. We observed varying levels and profiles of disablement (up to 7.8\%) among the respondents as well.

Before discussing our findings, the results must first be considered in the context of some potential study limitations. Firstly, we obtained our results only from primary care sites in Lagos Island, whose patients particularly in terms of social class may not be representative of most primary care practices; however, the healthcare centers in which the study was carried out share some other common features with many other centers in Nigeria. Secondly, there is the potential for the sources of error associated with studies involving subjects whose primary aims were to receive medical treatments rather than being interviewed for research purposes. Notwithstanding the above caveats, it should be emphasized that screening for psychiatric disorders in primary care is an important step to improving services. This should prompt physicians to consider the subsequent use of a full diagnostic interview and referral to specialized psychiatric services whenever necessary.

Our findings corroborate earlier ones indicating high prevalence of psychiatric morbidity in the primary care $[14,21]$. In this study it was found that the prevalence of psychiatric morbidity was $45.8 \%$. This figure seemingly appears higher than reported prevalence between $25 \%$ and $38 \%$ from some other countries of the world $[2,32,33]$. There is no simple explanation for this high figure, but a possible consideration may be the fact that this study was carried out in Lagos, a city with very difficult living and working conditions.

More striking still is the higher prevalence of psychiatric syndromes among these primary care service users as described by the ICD 10 PC as compared with the GHQ12. This suggests that ICD 10 PC captures some other mental health problems than the GHQ screens for. Such may include alcohol use disorder. This suggests that ICD 10 PC may be a more sensitive tool in screening for psychiatric morbidity in the primary care.

The pattern of symptom presentation and common mental disorders in this study are similar and comparable to other studies of mental health prevalence in primary health care. The disorders are depression, anxiety, difficulty in social functioning and disablement, sleep problems, Chronic fatigue, alcohol use problems, and unexplained somatic complaints. The findings correspond with World Health Organization's [34] report that the commonest problems identified among primary health care attendants were depression, anxiety, alcohol misuse, somatoform disorder, and neurasthenia.

We found a prevalence rate of $50.0 \%$ for depressive syndrome, a figure close to $51.7 \%$ (depressive neurosis) earlier reported in some parts of Nigeria. The prevalence of unexplained somatic complaint of $57.5 \%$ was higher than that of somatoform disorder (47.6\%) reported in an earlier study in Nigeria [35]. The high prevalence of unexplained somatic complaints reported in this study may not be explained as a reflection of the tendency of African patients to present their psychological distress with somatic symptoms as studies have shown that there is no cultural variation in somatization [36]. However, this high rate of somatization may suggest a pathway to more severe mental disorders. An interesting finding from this study is the higher than expected prevalence of disablement experienced by these patients $(2.0-7.8 \%)$ which is, to the best of our knowledge, the first estimate of prevalence of disability from psychosis in a primary care setting in Nigeria. This suggests the impact of psychosis on its sufferers in nonclinical population, in this instance primary care setting where the primary reason for service use is for general medical conditions.

The present study found certain sociodemographic correlates of psychiatric morbidity somewhat different from previous ones that found female gender, being unemployed, and being separated or divorced to be associated with a higher prevalence of psychiatric disorder $[1,2,4]$. The present study identified only young age, male gender, and being divorced to be associated with psychiatric morbidity.

A major problem of the estimates of psychiatric disorders in Nigeria is the choice of research methodology. Most psychiatric screening instruments were developed in countries outside Nigeria. In other words, there is a scarcity of published literature on different Nigeria languages.

The use of psychiatric screening scales in a language and culture other than that for which it was designed and developed may create considerable problems. This is because of the influence of translation and other sociocultural factors. Some researchers prefer to design new, culture-specific instruments. Ideally, a screening scale should be developed in the cultural setting in which it is to be used. It would, however, be irrational to ignore well-established instruments developed in other cultures since many phenomena and concepts are common between cultures. A critical, careful approach is, therefore, vital when translating, modifying, and validating psychiatric instruments before they are used across cultures. 


\section{Disclosure}

All authors agree to the construct of the paper.

\section{Conflict of Interests}

The authors declare that they have no conflict interests.

\section{Acknowledgments}

Acknowledgment is given to the Director of Ethical Review Board of The Lagos State Ministry of Health, for granting ethical approval to undertake this study. The assistance of Dr. A Akinnuoye, Dr. O Ayinde, Dr. O Ojediran, Sola Ogundeji and Christie Alabi in data collection is also appreciated. The authors acknowledge the funding support of New World Psychiatric Hospital, Ibadan.

\section{References}

[1] D. P. Goldberg, C. Kay, and L. Thompson, "Psychiatric morbidity in general practice and the community," Psychological Medicine, vol. 6, no. 4, pp. 565-569, 1976.

[2] J. E. Barrett, J. A. Barrett, T. E. Oxman, and P. D. Gerber, "The prevalence of psychiatric disorders in a primary care practice," Archives of General Psychiatry, vol. 45, no. 12, pp. 1100-1106, 1988.

[3] H. C. Schulberg, "Mental disorders in the primary care setting: research priorities for the 1990s," General Hospital Psychiatry, vol. 13, no. 3, pp. 156-164, 1991.

[4] E. Badamgarav, S. R. Weingarten, J. M. Henning et al., "Effectiveness of disease management programs in depression: a systematic review," American Journal of Psychiatry, vol. 160, no. 12, pp. 2080-2090, 2003.

[5] J. Halverson and C. Chan, "Screening for psychiatric disorders in primary care," Wisconsin Medical Journal, vol. 103, no. 6, pp. 46-51, 2004.

[6] S. L. Varma and M. Z. Azhar, "Psychiatric symptomatology in a primary health setting in Malaysia," Medical Journal of Malaysia, vol. 50, no. 1, pp. 11-16, 1995.

[7] S. Weich and G. Lewis, "Poverty, unemployment, and common mental disorders: population based cohort study," British Medical Journal, vol. 317, no. 7151, pp. 115-119, 1998.

[8] M. Ansseau, M. Dierick, F. Buntinkx et al., "High prevalence of mental disorders in primary care," Journal of Affective Disorders, vol. 78, no. 1, pp. 49-55, 2004.

[9] R. Araya, R. Wynn, R. Leonard, and G. Lewis, "Psychiatric morbidity in primary health care in Santiago, Chile. Preliminary findings," British Journal of Psychiatry, vol. 165, pp. 530 $533,1994$.

[10] M. Ansseau, B. Fischler, M. Dierick, A. Mignon, and S. Leyman, "Prevalence and impact of generalized anxiety disorder and major depression in primary care in Belgium and Luxemburg: the GADIS study," European Psychiatry, vol. 20, no. 3, pp. 229-235, 2005.

[11] K. Kroenke, R. L. Spitzer, J. B. W. Williams, P. O. Monahan, and B. Löwe, "Anxiety disorders in primary care: prevalence, impairment, comorbidity, and detection," Annals of Internal Medicine, vol. 146, no. 5, pp. 317-326, 2007.

[12] R. Agabio, M. Nioi, C. Serra, P. Valle, and G. L. Gessa, "Alcohol use disorders in primary care patients in Cagliari, Italy," Alcohol and Alcoholism, vol. 41, no. 3, pp. 341-344, 2006.
[13] B. Duran, M. Sanders, B. Skipper et al., "Prevalence and correlates of mental disorders among Native American women in primary care," American Journal of Public Health, vol. 94, no. 1, pp. 71-77, 2004.

[14] O. Gureje, O. O. Omigbodun, R. Gater, R. A. Acha, B. A. Ikuesan, and J. Morris, "Psychiatric disorders in a pediatric primary care clinic," British Journal of Psychiatry, vol. 165, pp. 527-530, 1994.

[15] M. J. L. Pedreira and G. E. Sardinero, "Prevalence of mental disorders in childhood in paediatric primary care," Actas LusoEspanolas de Neurologia, Psiquiatriay Ciencias Afines, vol. 24, no. 4, pp. 173-190, 2003.

[16] V. Eapen, M. Al-Sabosy, M. Saeed, and S. Sabri, "Child psychiatric disorders in a primary care Arab population," International Journal of Psychiatry in Medicine, vol. 34, no. 1, pp. 51-60, 2004.

[17] O. P. Almeida, O. V. Forlenza, N. K. Costa Lima et al., "Psychiatric morbidity among the elderly in a primary care setting-report from a survey in Sao Paulo, Brazil," International Journal of Geriatric Psychiatry, vol. 12, no. 7, pp. 728736, 1997.

[18] M. Olafsdottir, J. Marcusson, and I. Skoog, "Mental disorders among elderly people in primary care: the Linkoping study," Acta Psychiatrica Scandinavica, vol. 104, no. 1, pp. 12-18, 2001.

[19] S. Argyriadou, H. Melissopoulou, E. Krania, A. Karagiannidou, I. Vlachonicolis, and C. Lionis, "Dementia and depression: two frequent disorders of the aged in primary health care in Greece," Family Practice, vol. 18, no. 1, pp. 87-91, 2001.

[20] A. E. Danaci, G. Dinç, A. Deveci, F. S. Şen, and I. Içelli, "Postnatal depression in Turkey: epidemiological and cultural aspects," Social Psychiatry and Psychiatric Epidemiology, vol. 37, no. 3, pp. 125-129, 2002.

[21] O. A. Abiodun, "Postnatal depression in primary care populations in Nigeria," General Hospital Psychiatry, vol. 28, no. 2, pp. 133-136, 2006.

[22] S. K. Indran, "Current perspective on community psychiatry," Malaysian Journal of Psychiatry, vol. 2, pp. 90-94, 1994.

[23] M. P. Deva, "Psychiatry for the general practitioner," Medical Journal of Malaysia, vol. 52, no. 1, pp. 99-101, 1997.

[24] J. Scicchitano, P. Lovell, R. Pearce, J. Marley, and I. Pilowsky, "Illness behavior and somatization in general practice," Journal of Psychosomatic Research, vol. 41, no. 3, pp. 247-254, 1996.

[25] S. Pini, D. Berardi, P. Rucci et al., "Identification of psychiatric distress by primary care physicians," General Hospital Psychiatry, vol. 19, no. 6, pp. 411-418, 1997.

[26] R. L. Spitzer, J. B. W. Williams, K. Kroenke et al., "Utility of a new procedure for diagnosing mental disorders in primary care: the PRIME-MD 1000 study," Journal of the American Medical Association, vol. 272, no. 22, pp. 1749-1756, 1994.

[27] NPC. National population Commission, 1991 Census.

[28] The Research Advisors Sample Size Table, http://researchadvisors.com/.

[29] D. Goldberg and P. Williams, User's Guide to the General Health Questionnaire, NFER-Nelson, London, UK, 1988.

[30] World Health Organization, ICD 10 Mental Disorder Check List (Primary Care), http://whqlibdoc.who.int/hq/1998/WHO _MSA_MNHIEAC_98.1.pdf.

[31] SPSS. Statistical Package for Social Studies, Version 15.0, SPSS, Chicago, Ill, USA.

[32] L. G. Kessler, P. D. Cleary, and J. D. Burke, "Psychiatric disorders in primary care: results of a follow-up study," Archives of General Psychiatry, vol. 42, no. 6, pp. 583-587, 1985. 
[33] A. A. Noorbala, S. A. B. Yazdi, M. T. Yasamy, and K. Mohammad, "Mental health survey of the adult population in Iran," British Journal of Psychiatry, vol. 184, pp. 70-73, 2004.

[34] WHO World Mental Health Survey Consortium, "Prevalence, severity, and unmet need for treatment of mental disorders in the World Health Organization World Mental Health Surveys," Journal of the American Medical Association, vol. 291, no. 21, pp. 2581-2590, 2004.

[35] O. Ogunsemi, F. Oluwole, F. Abasiubong et al., "Detection of mental disorders with the Patient Health Questionnaire in primary care settings in Nigeria," Mental Illness, North America, vol. 2, no. 1, 2010.

[36] D. Bhugra and A. Mastrogianni, "Globalisation and mental disorders: overview with relation to depression," British Journal of Psychiatry, vol. 184, pp. 10-20, 2004. 


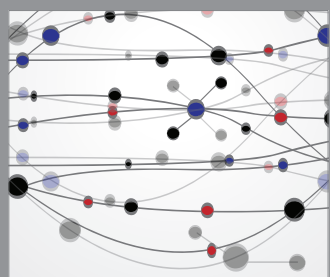

The Scientific World Journal
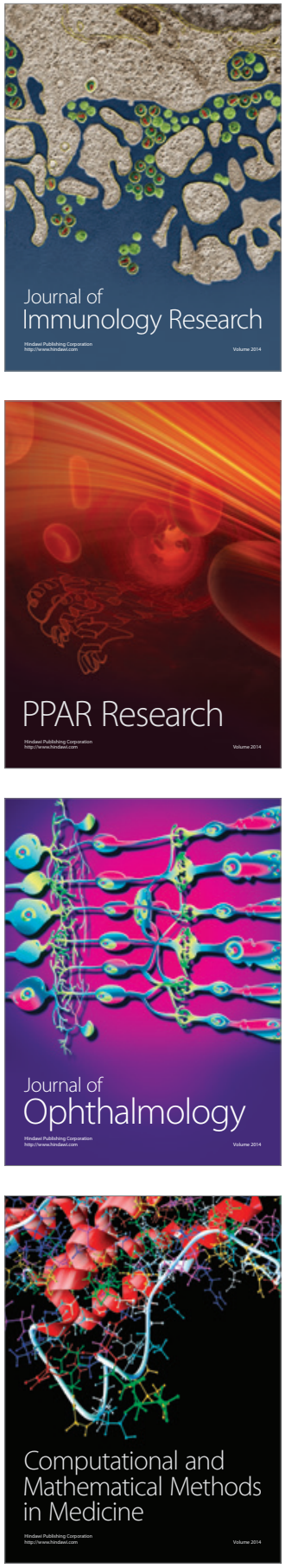

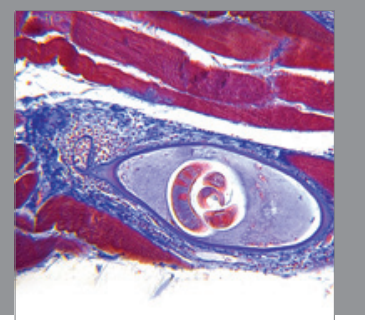

Gastroenterology

Research and Practice
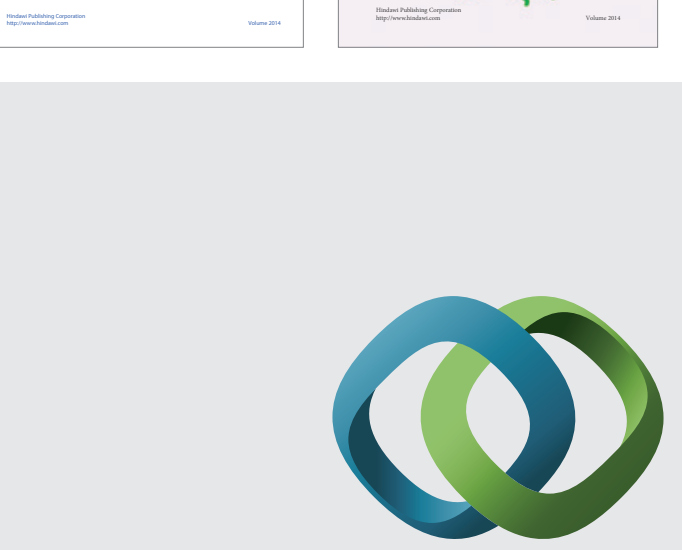

\section{Hindawi}

Submit your manuscripts at

http://www.hindawi.com
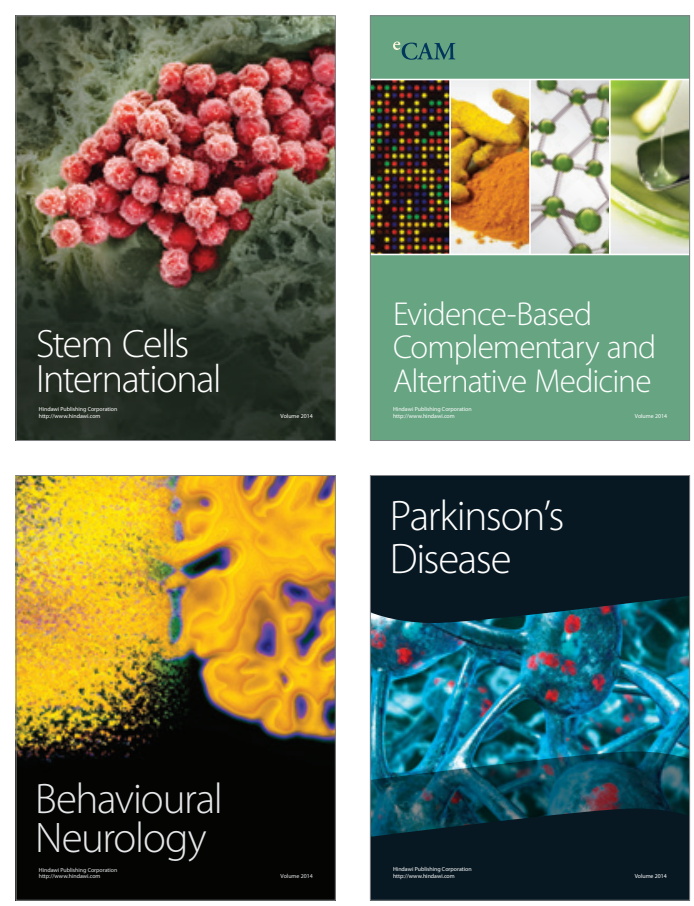

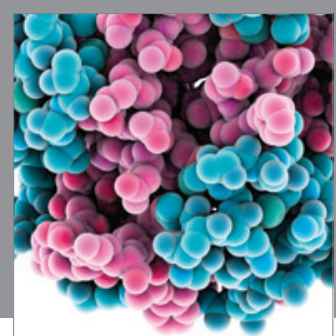

Journal of
Diabetes Research

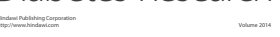

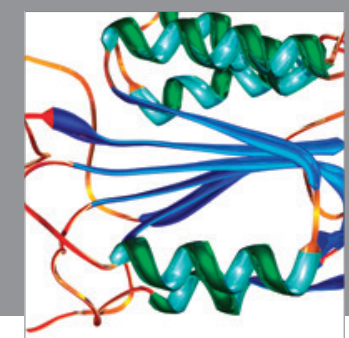

Disease Markers
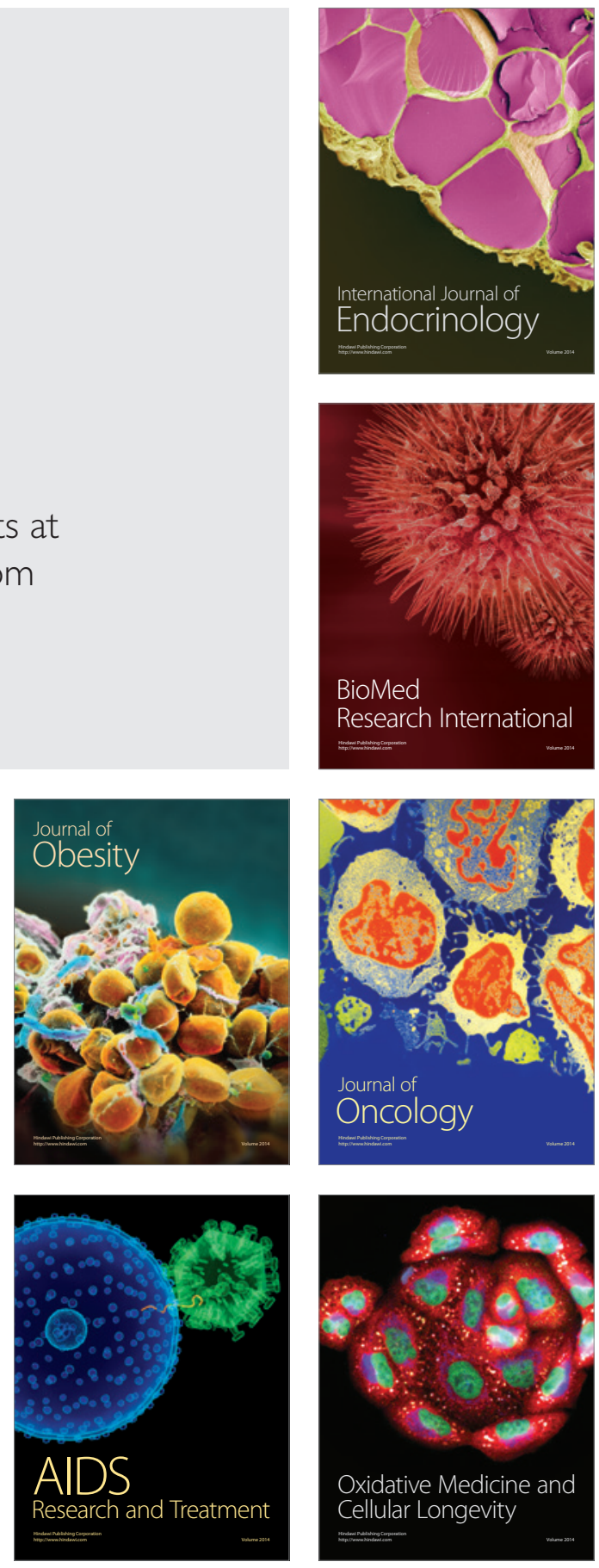